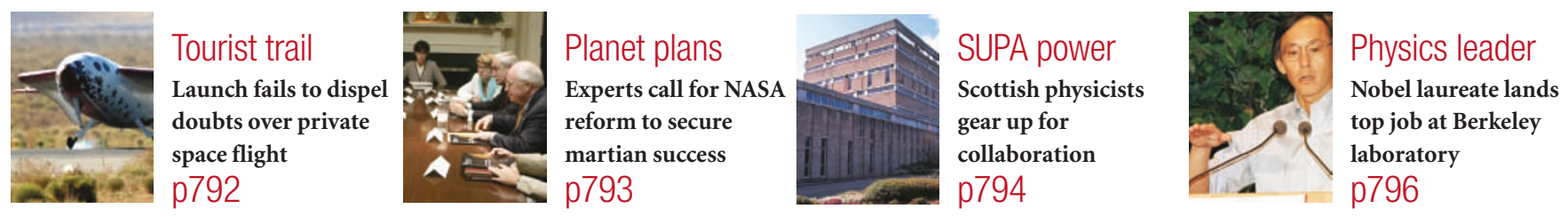

\title{
Biologists seek stamp of approval to send live fruitfiles by post
}

\section{David Cyranoski}

Biomedical researchers are not normally viewed as outlaws. But those who post live creatures, such as fruitflies (Drosophila), to their colleagues are currently breaking an international agreement.

So Drosophila distributors have launched a bid to get international postal regulations modified. If they succeed, it would be unambiguously legal to send live fruitflies through the post.

The bid is backed by the US Department of State and will be considered at this September's meeting of the Universal Postal Union (UPU) - the United Nations organization that administers global postal rules in Bucharest, Romania. Officials close to the process say they are confident that the amendment will be approved.

The contents of mailbags are coming under ever-closer scrutiny, and the need for fruitflies in the lab is on the rise. But many researchers and materials distributors around the world are a little hazy about the existing rules on sending live organisms through the post. The current Universal Postal Convention states that only bees, leeches, silkworms and "parasites and destroyers of noxious insects intended for control of those insects" are exempt from its bar on the international posting of live animals. Research organisms are out of luck, says Akhilesh Mathur, mail programme manager of the UPU, which administers the convention.

So Kevin Cook, who manages the Bloomington Drosophila Stock Center at Indiana University, is leading a drive to change the rules. He points out that Drosophila use is now central to biological and medical research worldwide, with close to 5,000 Drosophila-related papers published every year. Thousands of researchers have benefited from his centre's services, he says, including those at 421 laboratories in 38 countries to which the centre sent 43,000 fly cultures in 2003 alone. Cook sees the amendment as necessary to ensure that this trade can continue within international rules.

With the assistance of the state department, Cook is rallying support for the convention to pass an amendment that would add

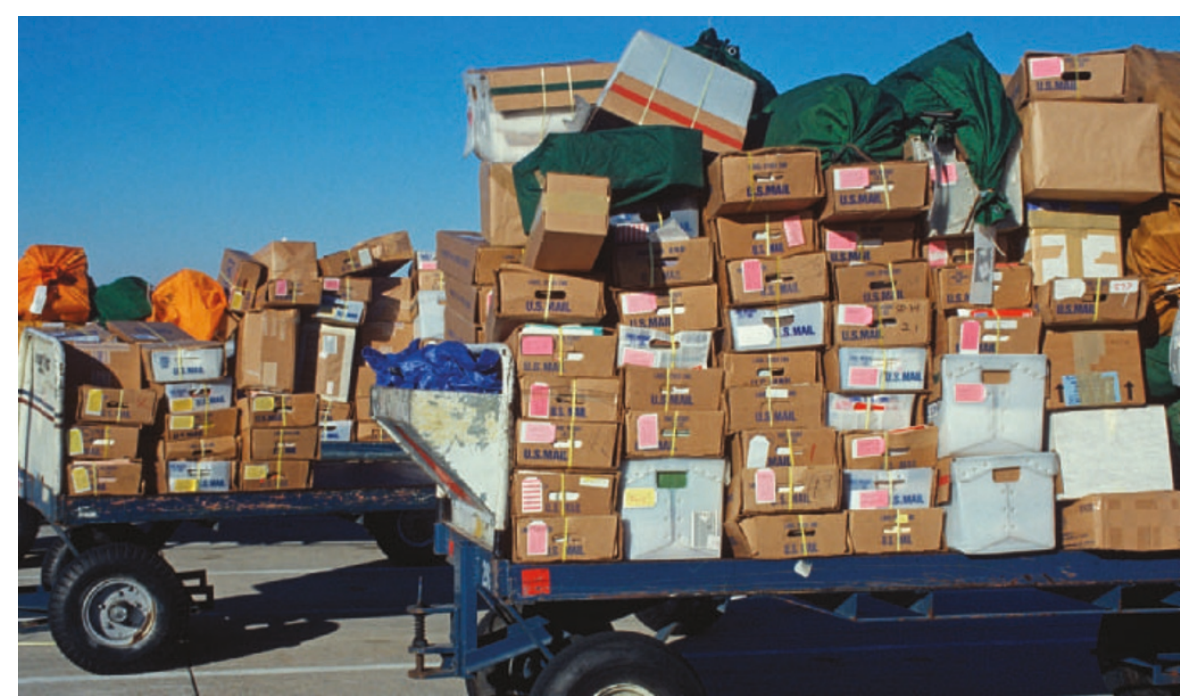

Mail orders: current international rules prohibit the transfer of most live animals by post.

"flies of the family Drosophilidae for biomedical research" to the current list of exemptions.

Although researchers have not complained much about the restriction - whose level of enforcement is difficult to establish - Cook says that it needs to be changed because authorities have been paying much closer attention to the precise contents of mail, especially in view of the bioterrorism scares that followed the terrorist attacks of 11 September 2001. The idea for the change arose in "the uncertain political context of mailing something that doesn't conform to postal regulations", he explains. Strict enforcement of the Cartagena Protocol on Biosafety, which sets restrictions on the shipment of genetically modified organisms, also threatens to restrict the movement of Drosophila (see Nature 428, 6; 2004).

\section{Delivery service}

But some scientists and distributors seem to think that posting Drosophila and other organisms is already allowed. The homepage for Kyoto Institute of Technology's Drosophila Genetic Resource Center, Japan's largest distributor, for example, states that "perishable biological substances" are exempt from postal restrictions.

And officials at the Caenorhabditis
Genetics Center at the University of Minnesota, a major international distributor of the nematode Caenorhabditis elegans, another ubiquitous model organism for research, say that they have experienced few difficulties when labelling the worms as "non-pathogenic, non-parasitic biological specimens of no commercial value" for the roughly 35 international shipments they make a week. "When the authorities ask for additional information we tell them exactly what's inside, and include publications showing their use in research," says Theresa Stiernagle, curator of the centre.

Mathur says that the proposal is likely to be passed by the convention, which only takes place every five years, in which case it would come into effect on 1 January 2006. But even if this happens, researchers say privately that the change could create headaches for shippers of other model organisms, by highlighting the fact that their shipment is against the rules.

If it fails, the implications could be severe for fruitfly researchers. Distributors might have to resort to the special shippers they already use for larger organisms, increasing their costs tenfold. In the worst case scenario, Cook says, "the stock centre would stop sending flies to anyone outside the United States because the costs would be too high". 